\title{
Especies de Agaricales (Basidiomycota) halladas por primera Vez en la Argentina: Agrocybe molesta, Coprinopsis ROMAGNESIANA Y GYMNOPUS VILLOSIPES
}

\author{
BERNARDO E. LECHNER ${ }^{1}$
}

\begin{abstract}
Resumen: Tres interesantes especies: Agrocybe molesta, Coprinopsis romagnesiana y Gymnopus vellosipes, fueron encontradas por primera vez en Argentina, en el Partido de Pinamar, Provincia de Buenos Aires. Las especies fueron descriptas e ilustradas.
\end{abstract}

Palabras clave: Nuevas citas, Omphalotaceae, Psathyrellaceae, Strophariaceae.

Summary: Species of Agaricales found for first time in Argentina: Agrocybe molesta, Coprinopsis romagnesiana and Gymnopus villosipes. Three interesting species: Agrocybe molesta, Coprinopsis romagnesiana and Gymnopus vellosipes were found for the first time in Argentina, Pinamar, Province of Buenos Aires. The species were described and illustrated.

Key words: New records, Omphalotaceae, Psathyrellaceae, Strophariaceae.

\section{INTRODUCCIÓN}

Durante un estudio sobre la biodiversidad de Agaricales en el Partido de Pinamar, Provincia de Buenos Aires, fueron halladas por primera vez en la Argentina tres interesantes especies: Agrocybe molesta (Lasch) Singer, Coprinopsis romagnesiana (Singer) Redhead, Vilgalys \& Moncalvo y Gymnopus villosipes (Cleland) Desjardin, Halling \& B.A. Perry.

El género Agrocybe en la Argentina ha sido estudiado por Uhart y se han encontrado, hasta ahora, 19 especies pertenecientes a los grupos $A$. cylindrica, A. praecox y A. pediales (Uhart, 2006). Agrocybe molesta pertenece al grupo de $A$. praecox $\mathrm{y}$, si bien se han encontrado representantes del mismo en la Argentina, A. molesta no había sido encontrada hasta el momento.

El género Gymnopus pertenece a la familia

\footnotetext{
1 Laboratorio de Micología, PROPLAME-PRHIDEBCONICET, Departamento de Biodiversidad y Biología Experimental, Facultad de Ciencias Exactas y Naturales, Universidad de Buenos Aires, 1428, CABA, Buenos Aires, Argentina, E-mail: blechner@bg.fcen.uba.ar.
}

Omphalotaceae y sólo han sido encontradas unas pocas especies hasta el momento en la Argentina (Niveiro \& Albertó, 2013). Gymnopus villosipes (Cleland) Desjardín, Hallin \& Perry es una especie encontrada comúnmente en la costa de California, EE.UU. (Desjardin et al., 1997) y fue descripta por primera vez como Marasmius villosipes por Cleland (1934) a partir de colecciones obtenidas en pinares de Australia.

Con respecto al género Coprinopsis P. Karst, creado en 1881 (Karst, 1881), este tuvo un aumento en el número de especies luego que Redhead et al. (2001) propusieron subdividir el género Coprinus en Coprinus sensu stricto (Agaricaceae), Coprinellus, Coprinopsis y Parasola, estos tres últimos dentro de la familia Psathyrellaceae, basados en caracteres moleculares. Coprinopsis romagnesiana es una especie rara que fue coleccionada en Europa y América y tiene características morfológicas que la relacionan con Coprinopsis atramentaria (Bull.) Redhead, Vilgalys \& Moncalvo, una especie tóxica que produce el síndrome coprínico cuando es ingerida con alcohol (Piqueras, 2011).

En el presente trabajo se proveen ilustraciones y se describen las colecciones halladas. 


\section{Materiales y Métodos}

Los especímenes fueron descriptos macroscópicamente, teniendo en cuenta su aspecto, el tipo, forma, tamaño y color del píleo, las laminillas y el pie. Los colores se anotaron de acuerdo con Rayner (1970). La cita de nombres de autores y taxones concuerdan con Kirk \& Ansell (1992). Las abreviaturas de herbario, según Holmgren et al. (1990).

Para la descripción microscópica se ha seguido un método de examinación estándar para Agaricales (Wright \&Albertó, 2002). Se hicieron cortes de la superficie del píleo para observar las hifas del revestimiento pileico, y de las laminillas, para describir los elementos más importantes, tales como tipo de esporas, tamaño, color, tipo de basidios, presencia o ausencia de cistidios y sus dimensiones. Se utilizaron los siguientes símbolos: $\mathrm{Q}=$ coeficiente entre el largo y el ancho de las esporas; $\mathrm{Q}_{x}=$ media de los valores de $\mathrm{Q} ; \mathrm{n}=$ número de esporas medidas. El medio usado para la observación de material fue $\mathrm{KOH}$ al $5 \%$ y floxina acuosa al $1 \%$.

Las colecciones fueron depositadas en el herbario micológico del Departamento de Biodiversidad y Biología Experimental de la Facultad de Ciencias Exactas y Naturales de la UBA (BAFC).

\section{Resultados y Discusión}

\section{Omphalotaceae}

Gymnopus villosipes (Cleland) Desjardin, Halling\& B.A. Perry, Mycotaxon64: 142 (1997).

Marasmius villosipes Cleland, Toadstools and Mushrooms and Other Larger Fungi of South Australia (Adelaide) 1: 166 (1934)

Píleo (Fig. 1A) 6-14 mm diám. castaño, convexo cuando joven, luego expandiéndose a plano, a veces deprimido, margen al principio incurvado, luego recurvado, estriado, higrófano, superficie glabra. Carne blanquecina, fina. Laminillas adnexas, subdistantes, 16-20, moderadamente anchas, hasta $3 \mathrm{~mm}$, convexas, color similar al píleo, algo más amarillento, borde pálido, más notorio cuando seco, con 2-3 series de lamélulas. Pie 30-45 x 1-2 mm, central, derecho a curvado, con base subbulbosa, velutinoso, castaño grisáceo, blanquecino en el ápice. Olor no distinguido. Esporada blanquecina.

Esporas (Fig. 2A) 6,1-8,8 x 3,2-4,4 $\mu \mathrm{m} \mathrm{Q}=1,7-2,3$, $\mathrm{Q}_{\mathrm{x}}=1,95, \mathrm{n}=22$, elipsoidales a subamigdaliformes, hialinas, pared delgada. Basidios (Fig. 2B) 23-32 x 6-7 $\mu \mathrm{m}$, claviformes, 4-esporados. Pleurocistidios ausentes. Queilocistidios (Fig. 2C) 13-32 x 4,4-12 $\mu \mathrm{m}$, claviformes, hialinos, pared delgada, a veces con el ápice de forma irregular. Trama himenoforal subparalela, hifas 3-7,5 $\mu \mathrm{m}$ diám., hialinas, generativas, fibuladas. Pileipellis (Fig. 2D) un cutis de hifas generativas, fibuladas, no gelatinosas, con células terminales erectas a suberectas, claviformes, lisas, no incrustadas, 32,2-34,4 x 4-5 $\mu \mathrm{m}$. Hifas del contexto del píleo (Fig. 2E) semejantes a las de la trama himenoforal. Stipitipellis (Fig. 2F) una tricodermis con fascículos hifales de 200-230 $\mu \mathrm{m}$ long. formados por hifas generativas, fibuladas, pared delgada, 3,3-4,4 $\mu \mathrm{m}$ diám.

Material estudiado. ARGENTINA, Prov. Bs. As., Partido de Pinamar, Cariló, 3709'32.31'S, 56 55'27.42” O, 5-IV-2007. Leg. B. E. Lechner. PIN 28. BAFC 52.367, sobre ramitas, en suelo de bosque implantado de Pinus maritimus.

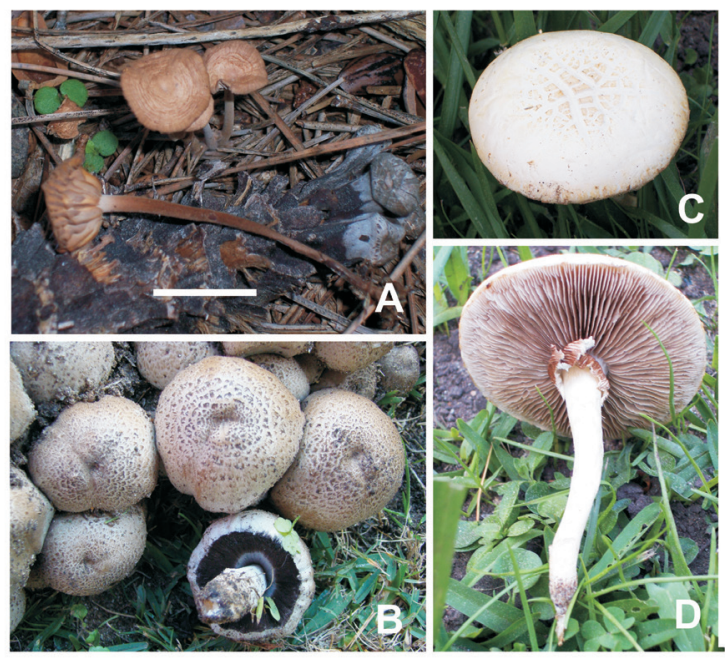

Fig. 1. Fotografías de los Basidiomas. A: Gymnospus villosipes; B: Coprinopsis romagnesiana; C y D: Agrocybe molesta. Barra de escala $=15 \mathrm{~mm}$ para A, $40 \mathrm{~mm}$ para B y $36 \mathrm{~mm}$ para C y D. 


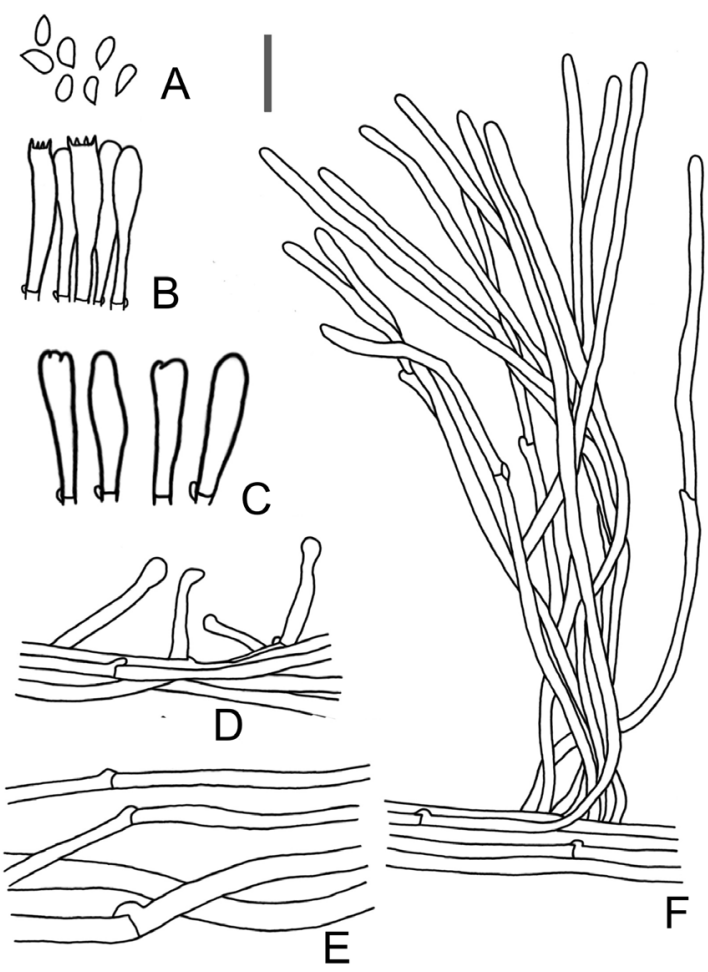

Fig. 2. Micromorfología de Gymnopus villosipes. A: Esporas; B: Basidios; C: Queilocistidios; D: Pileipellis; E: Hifas del contexto del píleo; F: Stipitipellis. Barra de Escala $=14 \mu \mathrm{m}$ para A-E, 20 $\mu \mathrm{m}$ para $\mathrm{F}$.

Observaciones. G. villosipes pertenece a la Sección Vestipedes debido a su pie velutinoso. Se caracteriza por tener un píleo castaño, laminillas concoloras y queilocistidios claviformes con el ápice irregular. Esta especie, nunca encontrada en la Argentina, seguramente amplió su distribución gracias al ambiente favorable encontrado en los bosques de pino, hábitat similar dónde fue encontrado en Australia, y Estados Unidos (Desjardin et al., 1997).

\section{Psathyrellaceae}

Coprinopsis romagnesiana (Singer) Redhead, Vilgalys \& Moncalvo, in Redhead, Vilgalys, Moncalvo, Johnson \& Hopple, Taxon 50(1): 230 (2001).

Coprinus romagnesianus Singer, Lilloa22: 459 (1951) [1949].
Coprinus atramentarius var. romagnesianus (Singer) Krieglst., Beitr. Kenntn. PilzeMitteleur.7: 63 (1991).

Píleo (Fig. 1B) 35-65 mm diám., 34-40 mm alto, ovoide campanulado a campanulado, en la madurez parabólico a plano, castaño claro, aclarándose hacia el margen hasta blanquecino, con numerosas escuámulas castaño-oscuras sobre la superficie que se diluyen desde el centro hacia el margen, a veces con escamas que se generan por craqueo de la superficie hacia el centro. Carne blanquecina, más ancha hacia el centro, 3-4 mm, afinándose hacia el margen. Laminillas libres, densas, anchas, hasta 13 $\mathrm{mm}$, blanquecinas a negras. Pie 55-80 x 10-15 mm, central, cilíndrico, liso, blanquecino. Olor fúngico. Esporada negra. Delicuescente en la madurez. Hábito cespitoso, a veces produciendo basidiomas masivamente, en grandes manojos.

Esporas (Fig. 3A) 8,0-11,3 x 5,3-6,7 $\mu \mathrm{m}$ $\mathrm{Q}=1,4-1,7, \mathrm{Q}_{\mathrm{x}}=1,55, \mathrm{n}=27$, elipsoidales $\mathrm{a}$ ovoides, castaño oscuras, pared gruesa, con poro germinativo. Basidios (Fig. 3B) 20,3-30 x 8,4-9,4 $\mu \mathrm{m}$, claviformes, 4-esporados, esterigmas 4,04,8 de largo, rodeados por 4-6 pseudoparáfisis, 19,6-20 x 14,4-17,5 $\mu \mathrm{m}$. Pleurocistidios (Fig. 3C) 96,2-127,8 x 17,0 -30 $\mu \mathrm{m}$, cilíndricos a utriformes. Queilocistidios (Fig. 3D) 41,1-68,1 x 16,5-29,0 $\mu \mathrm{m}$, similares a pleurocistidios. Trama himenoforal subparalela, formada por hifas de pared delgada, 3,4-8,9 $\mu \mathrm{m}$, que alternan con hifas conductoras,

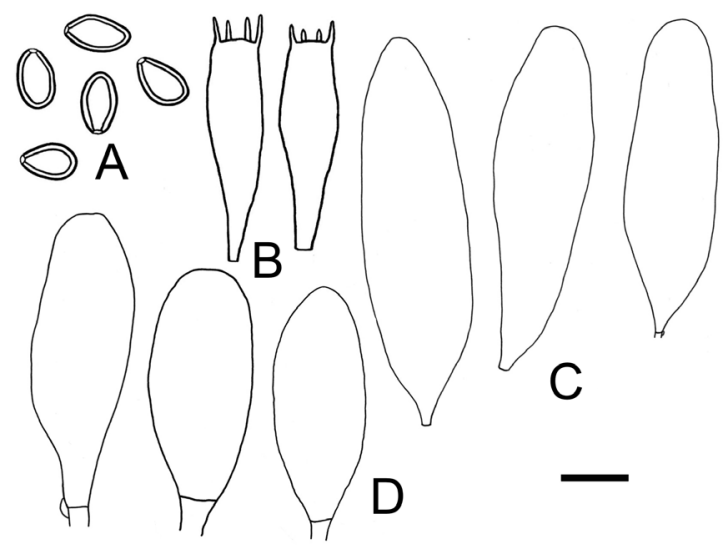

Fig. 3. Micromorfología de Coprinopsis romagnesiana. A: Esporas; B: Basidios; C: Pleurocistidios; D: Queilocistidios. Barra de escala= $12 \mu \mathrm{m}$ para A y B, $20 \mu \mathrm{m}$ para C y D. 
4-6,6 $\mu \mathrm{m}$ diám., fibuladas. Pileipellis un cutis formado por hifas generativas, pared delgada, fibuladas, 7,5-12,6 $\mu \mathrm{m}$ diám. Hifas del contexto del píleo 3,5-9,4 $\mu \mathrm{m}$ diám., fibuladas, alternando con hifas cortas, vesiculosas, 80,2 $\mu \mathrm{m}$ largo x $42 \mu \mathrm{m}$ ancho.

Material estudiado. ARGENTINA, Prov. Bs. As., Partido de Pinamar, Pinamar, 37 07'07.43" S 56 52'23.17' O, 15-VI-2008, Leg. B. E. Lechner, PIN 106, BAFC 52.366. Entre césped, cerca de Eucalyptus sp., rodeado de Santa Rita (Bougainvillea glabra).

Observaciones. Coprinopsis romagnesiana se caracteriza por tener un píleo ovoide campanulado a campanulado y escuámulas oscuras. Generalmente crece masivamente en grandes manojos cerca de árboles. C. romagnesiana tiene características morfológicas semejantes a Coprinopsis atramenaria (Bull.) Redehead Vilgalys \& Moncalvo, una especie tóxica cuando es ingerida con bebidas alcohólicas (Piqueras, 2011), pero diferenciándose por tener un píleo de tonalidades castañas con escuámulas, a diferencia de C. atramentaria que carece de ellas, y es de coloración grisácea. Es una especie rara, que fue encontrada en Europa y América (Gierczyk et al., 2011; Mešić \& Zzdenko Tkalčec, 2003; Sesli \& Denchev, 2014).

\section{Strophariaceae}

Agrocybe molesta (Lasch) Singer, Sydowia 30: 197 (1978).

Agaricus molestus Lasch, Linnaea 3: 421 (1828).

Agaricus molestus var. molestus Lasch, Linnaea 3: 421 (1828).

Agaricus molestus var. pseudosquamosus Lasch, Linnaea 3: 422 (1828).

Agrocybe molesta var. molesta (Lasch) Singer, Sydowia 30(1-6): 197 (1978) [1977].

Pholiota dura var. xanthophylla Bres., Fung. trident. 2(11-13): 52 (1892).

Agrocybe dura var. xanthophylla (Bres.) P.D. Orton, Trans. Br. mycol. Soc. 43(2): 174 (1960).

Agrocybe molesta var. xanthophylla (Bres.)

Bon \& Courtec., Docums Mycol. 18 (no. 69): 37 (1987).

Píleo (Fig. 1C, D) 4-8 cm diám., convexo cuando joven, luego expandiéndose a plano, blanquecino, desarrollando escamas en la madurez por craqueo de la superficie que se tornan castaño claras, pálidas. Laminillas adnexas, blanquecinas cuando jóvenes, luego castañas, apretadas. Pie 40-90 x 7-15 $\mathrm{mm}$, cilíndrico, liso, blanquecino, con un anillo delgado, membranáceo, superior. Carne blanca. Esporada castaña.

Esporas (Fig. 4A) 12,1-14,4 (-17,1) x 6,6-8,2 $\mu \mathrm{m} \mathrm{Q}=1,6-2,1, \mathrm{Q}_{\mathrm{x}}=1,80, \mathrm{n}=22$, elipsoidales, lisas, apenas truncadas, con poro germinativo. Basidios (Fig. 4B) 4-esporados, 28,8-31,7 x 8,1-10,5 $\mu \mathrm{m}$. Pleurocistidios no observados. Queilocistidios (Fig. 4C) 38,7-54,9 x 10,6-18,3 $\mu \mathrm{m}$. claviformes, hialinos, pared delgada. Trama himenoforal subparalela, hifas 3-7,8 $\mu \mathrm{m}$ diám. hialinas, generativas. Pileipellis (Fig. 4D) himeniforme, formada por elementos vesiculosos, 23,4-32,9 $\mathrm{x} 13,5-19,9 \mu \mathrm{m}$, con hifas del subcutis 3,0-5,7 $\mu \mathrm{m}$, hialinas, generativas. Hifas del contexto del píleo (Fig. 4E) 5,3-14,9 $\mu \mathrm{m}$, hialinas, generativas. Fíbulas presentes.

Material estudiado. ARGENTINA, Prov. Bs. As., Partido de Pinamar, Pinamar, 37 06'47.37" S 56 52’34.99 O, 15-X-2007. Leg. B. E. Lechner, PIN 89, BAFC 52368, al costado de calle, entre césped.

Observaciones. Agrocybe molesta se caracteriza por la presencia de escamas que se forman por
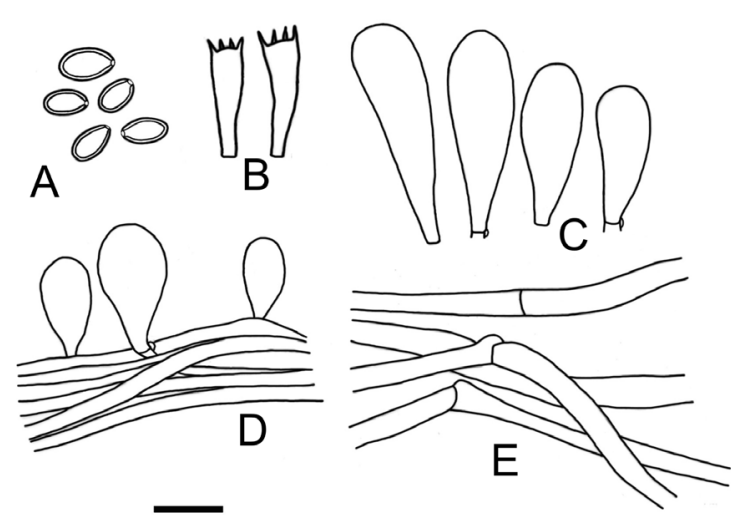

Fig. 4. Micromorfología de Agrocybe molesta. A: Esporas; B: Basidios; C: Queilocistidios; D: Pileipellis; E: Hifas del contexto del píleo. Barra de escala $=20 \mu \mathrm{m}$ para A-E. 
partición del píleo, anillo súpero y esporas de grandes dimensiones. Es además un descomponedor de los desechos que proviene del cortado del césped. Su posición taxonómica puede generar confusión, ya que A. molesta (Lasch) Singer es sinónimo de $A$. dura (Bolton) Singer según Watling (1982). A. dura (Bolton) Singer es una especie comestible y, exceptuando la confusión de la sinonimia de $A$. molesta, no se conoce ningún trabajo en el que se confirme la comestibilidad de esta última. Se diferencia de $A$. platensis por tener esta última esporas más chicas (10-12 x 6-7 $\mu \mathrm{m})$, una coloración diferente y carencia de escamas (Singer \& Digilio, 1951).

\section{Agradecimientos}

Deseo agradecer al CONICET y a la UBA por la financiación de este trabajo.

\section{Bibliografía}

CLELAND, J. B. 1934. Australian fungi: notes and descriptions No. 10. Trans. Royal Soc. S. Australia 58: 211-213.

DESJARDIN, E. E., R. E. HALLING \& B. P. PERRY. 1997. Gymnopus villosipes - a common collybioid agaric from California. Mycotaxon 64: 141-147.

GIERCZYK, B., A.KUJAWA, T. PACHLEWSKI, , A. SZCZEPKOWSKI \& M. WÓJTOW. 2011. Rare species of the genus Coprinus Pers. s. lato. Acta Mycologica 46: 27-73.

HOLMGREN, P. K., N. H. HOLMGREN \& L. C. BARNETT. 1990. Index Herbariorum. New York Botanical Garden, USA.

KARST, P. 1881. Hymenomycetes Fennici enumerati. Acta Societatis pro Fauna et Flora Fennica 2: 40 pp.

KIRK, P. M. \& A. E. ANSELL. 1992. Authors of fungal names. Index of Fungi, Suppl.
MEŠIĆ, A. \& Z. TKALČEC. 2003. Preliminary checklist of Agaricales from Croatia IV: families Bolbitiaceae, Coprinaceae, Entolomataceae and Pluteaceae, Mycotaxon 87: 283-309.

NIVEIRO, N. \& E. ALBERTÓ. 2012. Checklist of the Argentine Agaricales 4. Tricholomataceae and Polyporaceae. Mycotaxon 121: 499-500.

PIQUERAS J. 2011. Intoxicaciones por setas. En: MORÁN CHORRO, BALDIRÀ MARTÍNEZ DE IRUJO, MARRUECOS-SANT \& NOGUÉ XARAU (eds.), Toxicología Clínica, pp. 365-380. Difusión Jurídica y Temas de Actualidad S.A., Madrid.

RAYNER, R.W. 1970. A mycological colour chart. Commonwealth Agricultural Bureaux.

REDHEAD, S. A., R. VILGALYS, J. M. MONCALVO, J. JOHNSON \& J. S. HOPPLE. 2001. Coprinus Persoon and the disposition of Coprinus species sensu lato. Taxon 50: 203-241.

SESLI, E. \& C. M. DENCHEV. 2008. Checklists of the Myxomycetes, larger Ascomycetes, and larger Basidiomycetes in Turkey. Mycotaxon 106: 65.

SINGER, R. \& P. L. DIGILIO. 1951. Prodromo de la Flora Agaricina Argentina. Lilloa 25: 6-461.

UHART, M. 2006. Biodiversidad del género Agrocybe (Basidiomycetes, Agaricales) en la República Argentina. Estudios de filogenia molecular, compatibilidad sexual y cultivo intensivo del complejo Agrocybe cylindrica. Tesis. Universidad Nacional de General San Martín, San Martín, Prov. Buenos Aires.

WATLING, R. 1982. Bolbitiaceae: Agrocybe, Bolbitius\&Conocybe. En HENDRESON, D. M., P. D. ORTON \& R. WATLING (eds.), British Fungus Flora, Agarics and Boleti. Royal Botanical Garden, Edinburgh.

WRIGHT, J. E. \& E. ALBERTÓ. 2002. Guía de hongos de la región pampeana. I. Hongos con laminillas. L.O.L.A., Buenos Aires.

Recibido el 16 de marzo de 2015, aceptado el 11 de junio de 2015. 
